\title{
Kinetic effects on ion escape at Mars and Venus: Hybrid modeling studies
}

\author{
E. Kallio and R. Jarvinen \\ Finnish Meteorological Institute, Helsinki, Finland \\ (Received February 4, 2011; Revised June 26, 2011; Accepted August 11, 2011; Online published March 8, 2012)
}

\begin{abstract}
Kinetic effects are anticipated to play a role at Mars and Venus when the escape of planetary ions is considered because of the large ion gyroradius compared with the size of the planets. In this paper we have used the HYB hybrid model to analyze the role of kinetic effects at these non-magnetized planets by varying the mass of ions in the simulation. The test runs suggest that kinetic effects should be taken into account when properties and formation of the planetary three dimensional plasma and electromagnetic field environment are studied.
\end{abstract}

Key words: Mars, Venus, solar wind, ion escape, numerical simulation.

\section{Introduction}

Kinetic effects are anticipated to play a role around nonmagnetized celestial objects. At non-magnetized planets Mars and Venus the size of the solar wind interaction regions are small compared to the objects that have significant intrinsic magnetic fields, as is the situation at the Earth and at the giant planets Jupiter, Saturn, Uranus and Neptune. Moreover, the maximum strength of the magnetic field in the solar wind interaction region is much smaller at the nonmagnetized objects than near the Earth-like magnetized objects. Because of these characteristic features one may anticipate that, for example, the finite Larmor radius (FLR) effects, i.e., effects associated with ion gyro motion and nonMaxwellian velocity distributions of ions, affect the physics near Mars and Venus.

Some of the non-magnetized objects (such as the Moon and asteroids) have no atmosphere (or at most a very tenuous one). However, from the kinetic effects point of view, objects with an atmosphere are especially interesting because the solar wind can pick-up ionized planetary particles, such as $\mathrm{O}^{+}$and $\mathrm{O}_{2}{ }^{+}$ions from Mars and Venus. The ongoing plasma measurements at Venus (the Venus Express mission), at Mars (the Mars Express mission) and Titan (the Cassini mission) have increased noticeably our knowledge of plasma physical processes near these objects. An example of a manifestation of an FLR effect at Mars and Venus is the motion of planetary oxygen ions which are observed to be accelerated toward the direction of the convective electric field (see, for example, Luhmann et al., 2006; Barabash et al., 2007).

Another reason for the increasing interest in kinetic effects is the increased computer capacity, which enables three dimensional (3-D) kinetic plasma simulations in reasonable computational time. During the last ten years several 3-D hybrid models have been developed for and applied

Copyright (C) The Society of Geomagnetism and Earth, Planetary and Space Sciences (SGEPSS); The Seismological Society of Japan; The Volcanological Society of Japan; The Geodetic Society of Japan; The Japanese Society for Planetary Sciences; TERRAPUB.

doi:10.5047/eps.2011.08.014 to studies on how the solar wind interacts with various Solar System objects, such as Mercury, Venus, the Moon, Mars, Titan, asteroids and comets (see Ledvina et al., 2008, and references therein). In the hybrid simulation the positively charged ions are modeled as particles that are accelerated by the Lorentz force and possible other forces. Therefore, kinetic effects associated with FLR are automatically taken into account in the hybrid model.

The goal of this short paper is to summarize several plasma physical phenomena and features near Mars and Venus where kinetic effects are anticipated to play a role. The quantitative analysis is based on the HYB model. The paper is organized as follows. First the basic global modeling approaches are introduced. Then the HYB hybrid model is described and, finally, several plasma simulations are analyzed and their results presented.

\section{Global Numerical Models}

There are two different self-consistent global simulation approaches that are currently used to study how the solar wind interacts with Solar System objects, (1) Magnetohydrodynamic, MHD, simulations and (2) hybrid simulations (see, for example, Ledvina et al., 2008 for details). Most of the MHD models currently at use are multi-species MHD models where each ion species has its own densities calculated by continuity equations but all ions have the same bulk velocity and temperature. In a multi-fluid MHD model, instead, the motion of each ion species is calculated by its own momentum equation. Different MHD models can also differ in how they derive the electric field, E, from the magnetic field, $\mathbf{B}$, and the bulk velocity, $\mathbf{U}$. For example, in ideal MHD $\mathbf{E}=-\mathbf{U} \times \mathbf{B}$ and in resistive $\mathrm{MHD} \mathbf{E}=-\mathbf{U} \times \mathbf{B}+\eta \mathbf{j}$, which includes the electric current density $(\mathbf{j})$ and the resistivity $(\eta)$. The Hall MHD includes the Hall term $(\mathbf{j} \times \mathbf{B} /$ en $)$ in the electric field.

In a hybrid simulation positively charged ions are modeled as particles while electrons form a massless chargeneutralizing fluid. For example, if the Lorentz force is the dominating accelerating force then

$$
\mathrm{d} \mathbf{v}_{i} / \mathrm{d} t=\left(q_{i} / m_{i}\right)\left(\mathbf{E}+\mathbf{v}_{i} \times \mathbf{B}\right)
$$


where $q_{i}, m_{i}$, and $\mathbf{v}_{i}$ are the charge, mass and velocity of ion particles $i$. The ion velocity distribution can be arbitrary in all three dimensions while in MHD models it is assumed to be Maxwellian. The electric field is obtained from the electron momentum equation by assuming massless electrons (see, for example, Ledvina et al., 2008):

$$
\mathbf{E}=-\mathbf{U}_{\mathrm{e}} \times \mathbf{B}-\nabla\left(p_{\mathrm{e}}\right) / \rho_{\mathrm{e}}+\eta \mathbf{j}
$$

where $\mathbf{U}_{\mathrm{e}}$ is the electron bulk velocity, $p_{\mathrm{e}}$ is the electron thermal pressure and $\rho_{\mathrm{e}}$ is the electron charge density. The electron bulk velocity is obtained from the magnetic field and ion currents by using the Ampere's law without the displacement current:

$$
\begin{aligned}
& \mathbf{j}=\nabla \times \mathbf{B} / \mu_{\mathrm{o}}=-\rho_{\mathrm{e}} \mathbf{U}_{\mathrm{e}}+\Sigma_{i}\left(q_{i} n_{i} \mathbf{U}_{i}\right) \\
& \rightarrow \mathbf{U}_{\mathrm{e}}=\Sigma_{i}\left(q_{i} n_{i} \mathbf{U}_{i}\right) / \rho_{\mathrm{e}}-\nabla \times \mathbf{B} /\left(\mu_{\mathrm{o}} \rho_{\mathrm{e}}\right)
\end{aligned}
$$

Here the sum $\Sigma$ is over all ion particles $i$ and $n_{i}$ and $\mathbf{U}_{i}$ are ion densities and bulk velocities, respectively. In Eqs. (3a) and $(3 \mathrm{~b})$ the total electron density $\left(\rho_{\mathrm{e}}\right)$ is assumed to be equal to the total ion charge density $\left(=\Sigma_{i}\left(q_{i} n_{i}\right)\right)$, that is, plasma is assumed to be quasi-neutral.

It is worthy to note following issues concerning the roles of $q_{i}$ and $m_{i}$ in a hybrid model. First, the ion mass exists explicitly only in the Lorentz force (Eq. (1)) in the charge/mass ratio, $q_{i} / m_{i}$. If we, for example, change the mass of ions in a hybrid simulation run, changes in the simulation will result from Newton's second law (Eq. (1)). The ion charge, instead, exists explicitly in the equations of the hybrid model in two places, in (1) Newton's second law (Eq. (1)) and (2) the electron bulk velocity (Eq. (3b)). If the charge of ions is changed, changes in a simulation run are therefore due to the charge/mass ratio term in Eq. (1) and due to the charge which affects $\mathbf{E}$ via $\mathbf{U}_{\mathrm{e}}$ according to Eq. (3b).

In this brief paper we introduce several test runs performed to study kinetic effects in planetary plasma environments by using the HYB hybrid model family developed at the Finnish Meteorological Institute. Both Venusian and Martian plasma environments have previously been simulated with the HYB family models. Comprehensive details of the HYB versions for Mars (HYB-Mars) and Venus (HYB-Venus) can be found in Kallio et al. (2010), in Jarvinen et al. (2010a) and in references therein. Here we summarize only the basic features of the HYB models. The coordinate system in the HYB models is as follows: the $x$-axis points from the center of the planet to the Sun; the $z$-axis is perpendicular to the orbital plane of the planet pointing towards the angular momentum vector, and the $y$-axis completes the right hand coordinate system. The model grid is cube-shaped and centered at the planet's center. The planet and its atmosphere up to the exobase are modeled as a superconducting sphere, inside which the interplanetary magnetic field (IMF) cannot penetrate. An ion is taken away from the simulation if it hits the superconducting sphere. The grid spacing can increase with distance from the planet stepwise in planet-centric shells, the largest spacing being used in the volume between the outer boundary of the outermost shell and the cube-shaped exterior boundary of the grid. Such refined grid is used in the test runs presented in Section 3.4 while in all other presented runs the grid size was constant within the simulation box. All presented test runs include solar wind ions and also some planetary ions. The details of the input parameters are given below before the results of the runs are presented. The mass and charge of the ions in the HYB model can be chosen freely, which enables the study of the response of the solution to ion mass and charge.

\section{Kinetic Tests of Hybrid Model}

In this section we describe results from various hybrid model runs performed to study several kinetic effects. First, global plasma asymmetries are discussed (Section 3.1). Then kinetic effects associated with the newly formed ions are investigated, first motion of escaping planetary ions at Venus (Section 3.2) and then mass loading effects (Section 3.3). Finally kinetic effects related to the flowing solar wind plasma are studied by considering the role of the mass of the solar wind ions (Section 3.4). In all aforementioned cases one figure based on the HYB model is shown in order to illustrate the analyzed issue.

In the first two analyzed simulation runs (Figs. 1 and 2) upstream conditions are the nominal values at Venus. The ion density, ion velocity, ion temperature and the IMF are $14 \mathrm{~cm}^{-3}, 430 \mathrm{~km} \mathrm{~s}^{-1}, 10^{5} \mathrm{~K}$ and $\mathbf{B}=(-8.09,5.88,0) \mathrm{nT}$ $=\left(-\cos \left(36^{\circ}\right), \sin \left(36^{\circ}\right), 0\right) \times 10 \mathrm{nT}$, respectively.

\subsection{Global plasma and field asymmetries}

Martian and Venusian plasma environments include several asymmetries which affect the acceleration of planetary ions. Some of the asymmetries may also be of kinetic origin.

Both hybrid and MHD models generate hemispheric asymmetries if the IMF $x$-component is non-zero. If the IMF with a non-zero $x$-component is originally, say, on the $x y$-plane, then the morphology of the draped magnetic field lines are different on the dawn hemisphere $(y<0)$ and the dusk hemisphere $(y>0)$ (see, for example, Kallio et al., 2006 , figure 8 ). This asymmetry could be referred to as the (magnetic) dawn-dusk asymmetry. In an MHD model this asymmetry does not include kinetic effects while in a hybrid model kinetic effects contribute also to the dawn-dusk asymmetry. For example, part of the solar wind ions scatter back from the bow shock to the upstream solar wind on the hemisphere where the bow shock normal is parallel to the magnetic field and the foreshock ion beams are often observed both in the Earth and Mars.

Hybrid models give also rise to another hemispheric asymmetry which is usually referred to as the $\pm E_{\mathrm{sw}}$ asymmetry. Figure 1 gives an example of the $\pm E_{\text {sw }}$ asymmetry where the magnetic and electric fields are different on the so called " $+E_{\mathrm{sw}}$ hemisphere", where $E_{\mathrm{sw}}$ points away from the planet (in Fig. 1 in the $z>0$ hemisphere) and on the " $-E_{\mathrm{sw}}$ hemisphere", where $E_{\mathrm{sw}}$ points toward the planet (in Fig. 1 in the $z<0$ hemisphere). In Fig. 1 the IMF is $\left[B_{x}, B_{y}, B_{z}\right]=[-8.09,5.88,0] \mathrm{nT}$ but similar asymmetry exists also in runs which contains zero IMF $x$-component (see Zhang et al., 2010). This asymmetry can also be obtained in the Hall-MHD model but not in single-fluid or in multi-species MHD models. In the HYB model the magnitudes of $\mathbf{B}$ and $\mathbf{E}$ near the planet are stronger on the $+E_{\mathrm{sw}}$ 

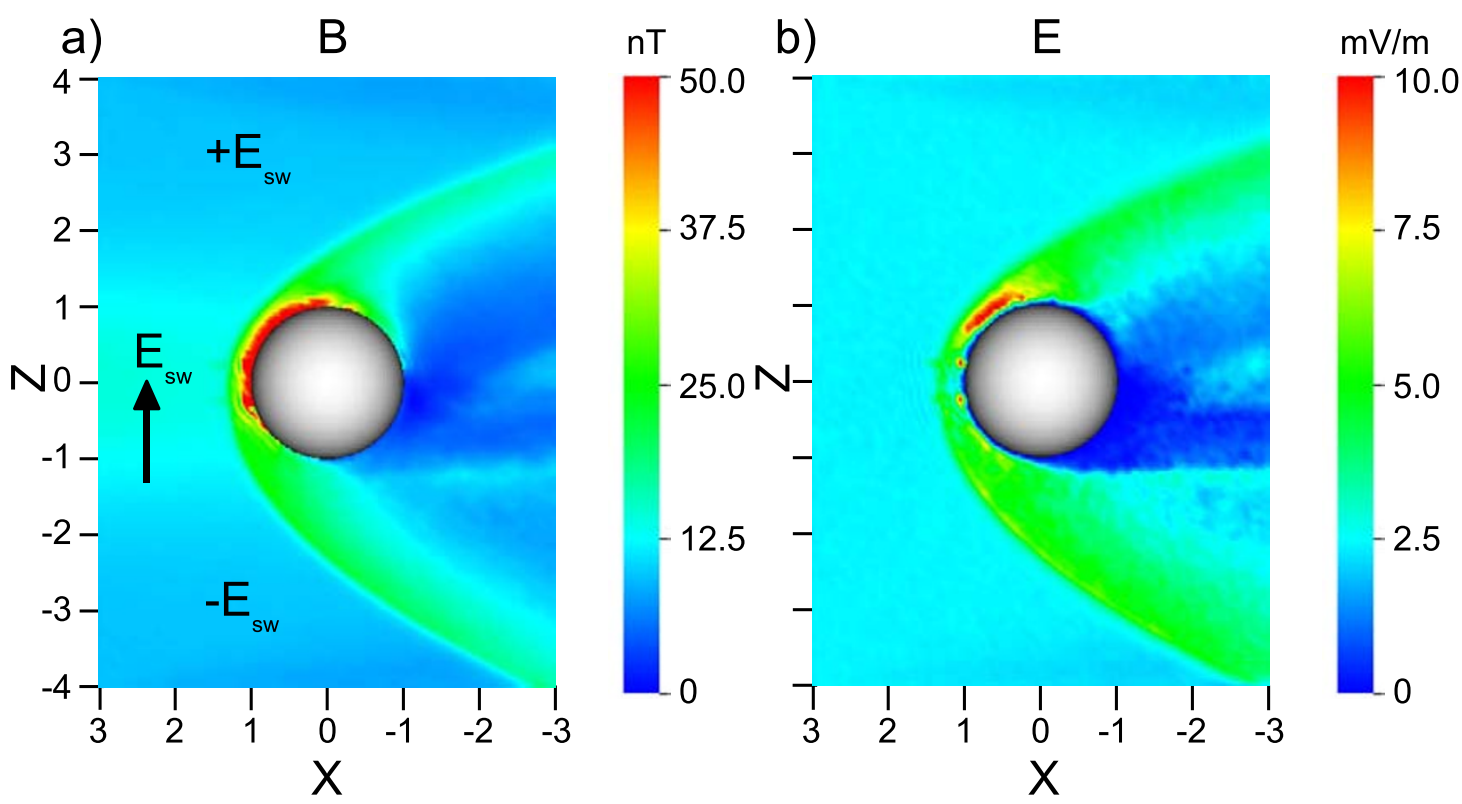

Fig. 1. A HYB-Venus run to study the Venusian electromagnetic environment: (a) the total magnetic field, (b) the total electric field on the noon-midnight plane $(y=0)$. The solar wind flow is coming from the left. The spherical shell illustrates Venus. See Jarvinen et al. (2010b; Run 4), for the details of the run. The unit of the axes is the radius of Venus.

hemisphere than on the opposite hemisphere (Fig. 1(a) and (b)). The asymmetry can also be seen in the HYB simulation when the draping of the magnetic field lines on the $+E_{\mathrm{sw}}$ and $-E_{\mathrm{sw}}$ hemispheres are analyzed. Such asymmetric field line draping has also been detected in the VEX magnetometer data (Zhang et al., 2010).

\subsection{The motion of escaping planetary ions}

Ions above the planetary exobase are accelerated to energies exceeding the escape energy by the Lorentz force and, therefore, it can be expected that global asymmetries seen in the Martian and Venusian electromagnetic field (c.f. Fig. 1) result in asymmetric ion escape patterns. It is well established that in the HYB-model the large ion gyroradii of planetary $\mathrm{O}^{+}$and $\mathrm{O}_{2}{ }^{+}$ions result in a situation where the heavy planetary ions originating near the planet are accelerated toward the $+E_{\mathrm{sw}}$ hemisphere due to the convective electric field at Mars (see, e.g. Kallio et al., 2010) and at Venus (Jarvinen et al., 2010a). Recently, more general interest in how the different $m / q$ ratio of planetary ions affects the escape pattern has, however, arisen due to the ASPERA-4 $\mathrm{H}^{+}$and $\mathrm{O}^{+}$observations at Venus (Barabash $e t$ al., 2007).

In Fig. 2 the role of the $m / q$ ratio is studied by test particle simulation where the electromagnetic field is obtained from the HYB-Venus model (see Jarvinen et al., 2010a, about the details of the used Venus run). In Fig. 2 test particles were launched from a spherical shell at $r=1.2 R_{\mathrm{V}}$ $\left(R_{\mathrm{V}}=6051.8 \mathrm{~km}\right)$ with an initial velocity of $1 \mathrm{~km} \mathrm{~s}^{-1}$ upwards. Four different ion masses were tested, proton mass $m_{\mathrm{p}}, 4 m_{\mathrm{p}}, 16 m_{\mathrm{p}}$ and $32 m_{\mathrm{p}}$, which could represent, for example, $\mathrm{H}^{+}, \mathrm{He}^{+}, \mathrm{O}^{+}$and $\mathrm{O}_{2}{ }^{+}$ions, respectively. One may anticipate that any asymmetric effect that becomes more evident for larger $m / q$ ratio indicates that FLR effect plays a role.

Comparison of Fig. 2(a)-2(d) suggests that the motion of planetary ions depends strongly on the ion mass. It is worth noting that the analyzed run contains a non-zero IMF $x$ component, which results in a strong asymmetry between the dawn side $(y<0)$ and the dusk side $(y>0)$ so that the ions move toward the dawn side because of the $\mathbf{E} \times \mathbf{B}$ drift motion, $\mathbf{V}_{\mathbf{E} \times \mathbf{B}}$. This is clearly seen in the $x y$-projection (Fig. 2, middle columns). One can also recognize that ion trajectories of the light ions $\left(m_{\mathrm{p}}\right.$ and $\left.4 m_{\mathrm{p}}\right)$ and the heavy ions $\left(16 m_{\mathrm{p}}\right.$ and $\left.32 m_{\mathrm{p}}\right)$ differ substantially. The spatial distribution of these ions are, therefore, quite different near Venus, the Venusian magnetosphere acting much like an ion "mass filter". It is worthy of emphasis that different ion trajectories are an FLR effect, and the trajectories of the magnetized light ions $\left(m_{\mathrm{p}}\right.$ and $\left.4 m_{\mathrm{p}}\right)$ follow in the first approximation $\mathbf{V}_{\mathbf{E} \times \mathbf{B}}$ motion while the heavy ions $\left(16 m_{\mathrm{p}}\right.$ and $32 m_{\mathrm{p}}$ ) are practically non-magnetized and do not follow the $\mathbf{V}_{\mathbf{E} \times \mathbf{B}}$ motion (Jarvinen et al., 2010a).

\subsection{Mass loading effects}

Physical effects associated with the addition of new ions into the solar wind have historically received much attention in cometary physics (see, for example, Omidi and Winske, 1987) and also when the particle and field data from Mars and Venus were interpreted. A hybrid simulation provides a modeling tool where various mass loading effects can be studied in detail because the ion velocity distribution function can be arbitrary in 3-D and there are no pre-assumptions about how the velocity of different ion species are correlated. Therefore, the velocity distributions of the newly formed ions can form a "ring" or a "shell", there can be several particle populations for the same ion species (for example, solar wind protons and $\mathrm{H}^{+}$photoions) and different ion species can move to different directions. In the hybrid model ion motions are self-consistently related to the electromagnetic field enabling studies of how the kinetic effects affect the magnetic and electric fields near the object, for example, near a comet, Mars or Venus.

Figure 3 shows an example of a test run where the mass 

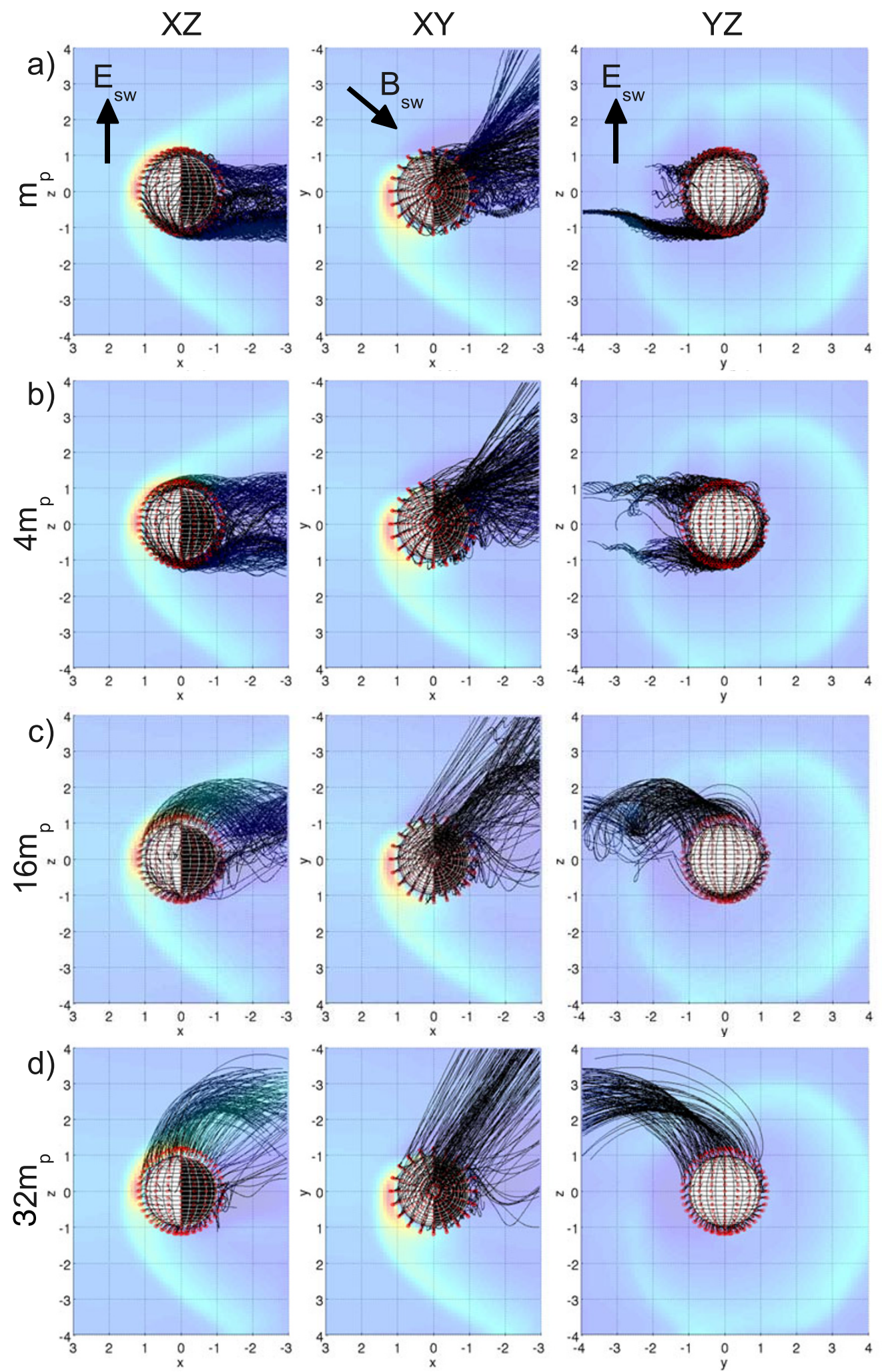

Fig. 2. Trajectories of test particles propagated in the global electric and magnetic fields from the HYB-Venus simulation run. Different particle masses were used to study finite Larmor radius effects: (a) proton mass $m_{\mathrm{p}}$, (b) $4 m_{\mathrm{p}}$, (c) $16 m_{\mathrm{p}}$ and (d) $32 m_{\mathrm{p}}$. All ions have a positive charge of one elementary charge (e). Three different projections are shown: $x z$ (left column), $x y$ (center column) and $y z$ (right column). The background coloring illustrates the magnetic field magnitude at the $y=0, z=0$ and $x=-2 R_{\mathrm{V}}$ planes. The particles were launched from a spherical shell at $r=1.2 R_{\mathrm{V}}$. The unit of the axes is the radius of Venus.

loading effects were studied with the HYB model. The presented test was designed to minimize any other effects than mass-loading. Therefore, the simulation does not include a solid object, as Mars or Venus, but only a neutral corona from where ions $\left(\mathrm{X}^{+}\right)$were generated. The ion production rate was assumed to have $1 / r^{2}$ dependence from the origin of the simulation at $(0,0,0)$. The total ion production rate within the simulation box was $10^{26} \mathrm{~s}^{-1}$. The new ions have 
a small initial temperature of $6000 \mathrm{~K}$. The size of the simulation box was $-18,000 \mathrm{~km}<x<18,000 \mathrm{~km},-24,000 \mathrm{~km}$ $<y, z<24,000 \mathrm{~km}$ and new ions are generated within that volume. The new ions affect the incident undisturbed solar wind by decreasing its velocity and deviating its direction. The strength of the mass loading effect in a given solar wind situation depends on the production rate of new ions and the mass of ions. Figure 3 was chosen to illustrate an example when the mass loading strongly deflects the solar wind flow. The mass of new ions, $\mathrm{X}^{+}$, added into the solar wind was assumed to be artificially high, 100 times the mass of $\mathrm{O}^{+}$ ions, and Fig. 3 gives a snapshot of the simulation at $140 \mathrm{~s}$ after the solar wind enters in the simulation box. The solar wind density, velocity and the IMF were chosen to be $10 \mathrm{~cm}^{-3}, 430 \mathrm{~km} \mathrm{~s}^{-1}$ and $(0,5.88,0) \mathrm{nT}$, respectively.

Figure 3 shows how the solar wind and newly formed ions have an opposite velocity with respect to the direction of the convective electric field, $\mathbf{E}_{\mathrm{sw}}=\left(0,0,\left|\mathbf{E}_{\mathrm{sw}}\right|\right)$, in the undisturbed solar wind: The newly formed $\mathrm{X}^{+}$ions move to $+\mathbf{E}_{\mathrm{sw}}$ while the decelerated solar wind $\mathrm{H}^{+}$ions move toward $-\mathbf{E}_{\mathrm{sw}}$. This is an FLR effect where the fast moving $\mathrm{H}^{+}$ions feel the Lorentz force that points toward $-\mathbf{E}_{\mathrm{sw}}$ while the slowly moving $\mathrm{X}^{+}$ions feel the Lorentz force toward $+\mathbf{E}_{\mathrm{sw}}$ direction. This affects the velocity of the ions because $\mathrm{X}^{+}\left(\mathrm{H}^{+}\right)$ions gain(loose) energy while moving along(against) the convective electric field. One way to see why ions move in those directions is to analyze the motion of ions in the electron bulk frame of reference, $\mathbf{U}_{\mathrm{e}}$, where the convective electric field is zero. Part of the electrons is associated with the fast flowing $\mathrm{H}^{+}$ions and part with the slowly moving $\mathrm{X}^{+}$ions. The magnitude of $\mathbf{U}_{\mathrm{e}}$ is therefore smaller than in the undisturbed solar wind where $\mathbf{U}_{\mathrm{e}}=$ $\mathbf{U}_{\mathrm{sw}}=\mathbf{U}\left(\mathrm{H}^{+}\right)$. In other words, in the moving $\mathbf{U}_{\mathrm{e}}$ frame of reference, $\mathrm{H}^{+}$ions are moving toward the $-x$ direction and rotating along $\mathbf{B}$ downward to $-z$ direction due to the $\mathbf{v} \times \mathbf{B}$ force (positive ions are rotating around $\mathbf{B}$ according to the left hand rule). The slowly moving $\mathrm{X}^{+}$ions are, instead, moving in the $\mathbf{U}_{\mathrm{e}}$ frame of reference to $+x$ direction and the $\mathbf{v} \times \mathbf{B}$ force accelerates these ions to $+z$ direction (the left hand rule).

The formation of the magnetic asymmetry between the $+E_{\mathrm{sw}}(z>0)$ and $-E_{\mathrm{sw}}$ hemispheres $(z<0)$ in Fig. 3(c) can also be understood by analyzing the motion of electrons: Fig. 3(c) is due to asymmetry in the electron motion (because of the frozen-in condition). When the role of the electron pressure and the magnetic diffusion is small (e.g., soon after new ions are formed and they have yet disturbed noticeably the solar wind flow), the magnetic field is "frozen in" to the electrons in the hybrid model because the model assumes then that $\mathbf{E}=-\mathbf{U}_{\mathrm{e}} \times \mathbf{B}$ (c.f. Eq. (2)). The magnetic flux tubes from the solar wind become therefore "pushed" toward $-z$ direction (see Fig. 3(b)) and this compression manifests itself as increasing magnetic field magnitude on the $z<0$ hemisphere (Fig. 3(b)). How the system develops and which stationary solution it finally reaches, depends on the details of the mass loading and on the properties of the solar wind.

Analysis of the effects of the mass loading, and the resulting electromagnetic asymmetries, at Mars and Venus are more difficult to perform than the test seen in Fig. 3 because the planets form impenetrable surfaces and have conducting ionospheres. A planetary ionosphere forms an effective obstacle against the solar wind flow which, in turn, forms a bow shock. Moreover, part of the solar wind protons flows around the planets while some of the protons can hit the atmosphere and become fast hydrogen atoms. For example, one may pose a question: Does the strongest magnetic field on the $+E_{\mathrm{sw}}$ hemisphere exist, because the magnetic flux tubes that move toward $-E_{\mathrm{sw}}$ direction are strongly "packed up" against the obstacle on the $+E_{\mathrm{sw}}$ hemisphere? A detailed analysis of such situation is, however, beyond of the scope of the current paper.

\subsection{The role of the mass of the solar wind ions}

In the next two sections the kinetic effects associated with the solar wind ions are studied. In the first presented analysis two simplified Mars runs were compared which have different mass of the solar wind ions $\left(m_{\text {ion }}\right)$, different densities $\left(n_{\mathrm{sw}}\right)$ and temperatures $\left(T_{\mathrm{sw}}\right)$. In the nominal run the solar wind includes protons $\left(m_{\text {ion }}=m_{\mathrm{p}}\right), n_{\mathrm{sw}}=2.7$ $\mathrm{cm}^{-3}$ and $T_{\mathrm{sw}}=15 \times 10^{4} \mathrm{~K}$. In the artificial test run these parameters were $m_{\text {ion }}=1 / 3 \times m_{\mathrm{p}}, n_{\mathrm{sw}}=3 \times 2.7 \mathrm{~cm}^{-3}$ and $T_{\mathrm{sw}}=1 / 3 \times 15 \times 10^{4} \mathrm{~K}$. In both runs $\mathbf{U}_{\mathrm{sw}}=(-485,0,0)$ $\mathrm{km} \mathrm{s}^{-1}$ and $\mathbf{B}_{\mathrm{sw}}=(0,2,0) \mathrm{nT}$. This means that in both runs the mass density and, consequently, the dynamic pressure $\left(=0.5 \times n_{\mathrm{sw}} \times m_{\mathrm{sw}} \times U_{\mathrm{sw}}^{2}\right)$ of the solar wind were identical. Moreover, the sound speed, $v_{\mathrm{s}}\left(\equiv \sqrt{\gamma k T / m_{i}}\right)$, where $\gamma$ is the adiabatic index) and the Alfvén speed of the solar wind, $v_{\mathrm{A}}\left(\equiv B / \sqrt{\mu_{\mathrm{o}} m_{i} n}\right)$, were also kept unchanged. Therefore, in both runs the upstream Alfvén and sonic Mach numbers are identical. In both runs the size of the simulation box was $-4 R_{\mathrm{M}}<x, y, z,<4 R_{\mathrm{M}}$. The simulation included three grid sizes: $0.2 R_{\mathrm{M}}$ at $r>3 R_{\mathrm{M}}, 0.1 R_{\mathrm{M}}$ at $2 R_{\mathrm{M}}<$ $r<3 R_{\mathrm{M}}$ and $0.05 R_{\mathrm{M}}$ at $r<2 R_{\mathrm{M}}$ where $r$ is the distance from the center of Mars. In the test runs Martian planetary ion environment was simplified in order to minimize the number of effecting factors and, therefore, planetary ions were assumed to contain only $\mathrm{O}^{+}$ions which are emitted at the exobase at the total rate of $1.4 \times 10^{25} \mathrm{O}^{+}$ions s${ }^{-1}$.

One may anticipate several differences between these two runs. First, gyroradii of the solar wind ions are three times larger in the $m_{\text {ion }}=m_{\mathrm{p}}$ case than in the $m_{\text {ion }}=$ $1 / 3 \times m_{\mathrm{p}}$ case if the ions move at similar velocity in similar magnetic field. In that respect the $1 / 3 \times m_{\mathrm{p}}$ run may therefore result in a more "Venus-like" situation, because the gyroradius/diameter of the planet ratio decreases (see, for example, Ledvina et al., 2008, for detailed discussion of the gyroradii at Mars and Venus). Secondly, if $m_{\text {ion }}=1 / 3 \times m_{\mathrm{p}}$ ion which starts at rest moves the length $L$, say, along the electric field $\mathbf{E}$, its velocity, $v_{i}$, is $\sim 1.7(=\sqrt{3})$ times larger compared to the $m_{\text {ion }}=m_{\mathrm{p}}$ ion which are somehow forced to make the identical trajectory $\left(e \times L \times E=0.5 \times m_{\text {ion }} \times v_{i}^{2}\right)$. This may affect the strength of how ions are heated at the bow shock and in the magnetosheath. Third, the charge density of the solar wind, $q \times n$, is larger in the $m_{\text {ion }}=1 / 3 \times m_{\mathrm{p}}$ run than in the $m_{\text {ion }}=m_{\mathrm{p}}$ run. This may affect the strength of the currents and, consequently, the associated magnetic field.

Figure 4 shows a comparison of the magnetic field in the $m_{\mathrm{p}}$ and $1 / 3 \times m_{\mathrm{p}}$ runs. Both runs result in a bow shock and an enhanced magnetic field (so called magnetic 


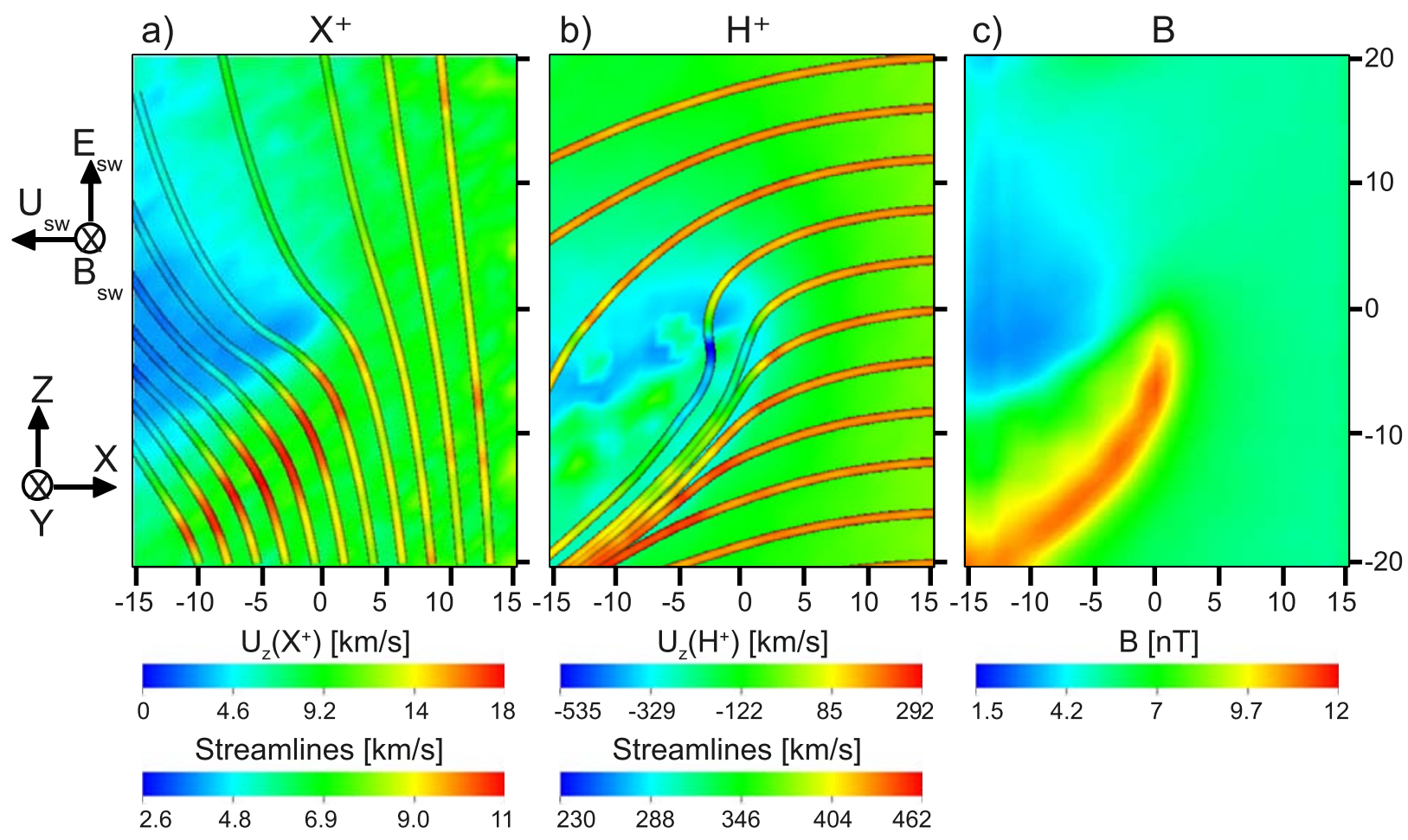

Fig. 3. Mass loading effects. The color on the $y=0$ plane show (a) the $U_{z}$ of newly ionized ion by e.g. photo ionization $\left(\mathrm{X}^{+}\right)$, (b) the $U_{z}$ of the solar wind $\mathrm{H}^{+}$ions and (c) the magnitude of the magnetic field. The tubes in (a) and (b) show the flow lines of $\mathrm{X}^{+}$and $\mathrm{H}^{+}$ions, respectively. The color on the surface of the tubes shows the total ion velocity. The direction of the undisturbed solar wind vector $\left(\mathbf{U}_{\mathrm{sw}}=(-430,0,0) \mathrm{km} \mathrm{s}{ }^{-1}\right)$, the IMF vector $\left(\mathbf{B}_{\mathrm{sw}}=(0,5.88,0) \mathrm{nT}\right)$ and the convective electric field $\left(\mathbf{E}_{\mathrm{sw}}=-\mathbf{U}_{\mathrm{sw}} \times \mathbf{B}_{\mathrm{sw}}\right)$ are given on the left. Note that the bulk flow of $\mathrm{X}^{+}$ions is toward the $+\mathbf{E}_{\mathrm{sw}}$ direction while $\mathrm{H}^{+}$ions move toward the $-\mathbf{E}_{\mathrm{sw}}$ direction. Note also that $\mathrm{X}^{+}$ions are produced in all places within the simulation box. The center of the $\mathrm{X}^{+}$ion source is at the center of images at $(x, y, z)=(0,0,0)$ and the unit of axes is $1,000 \mathrm{~km}$.
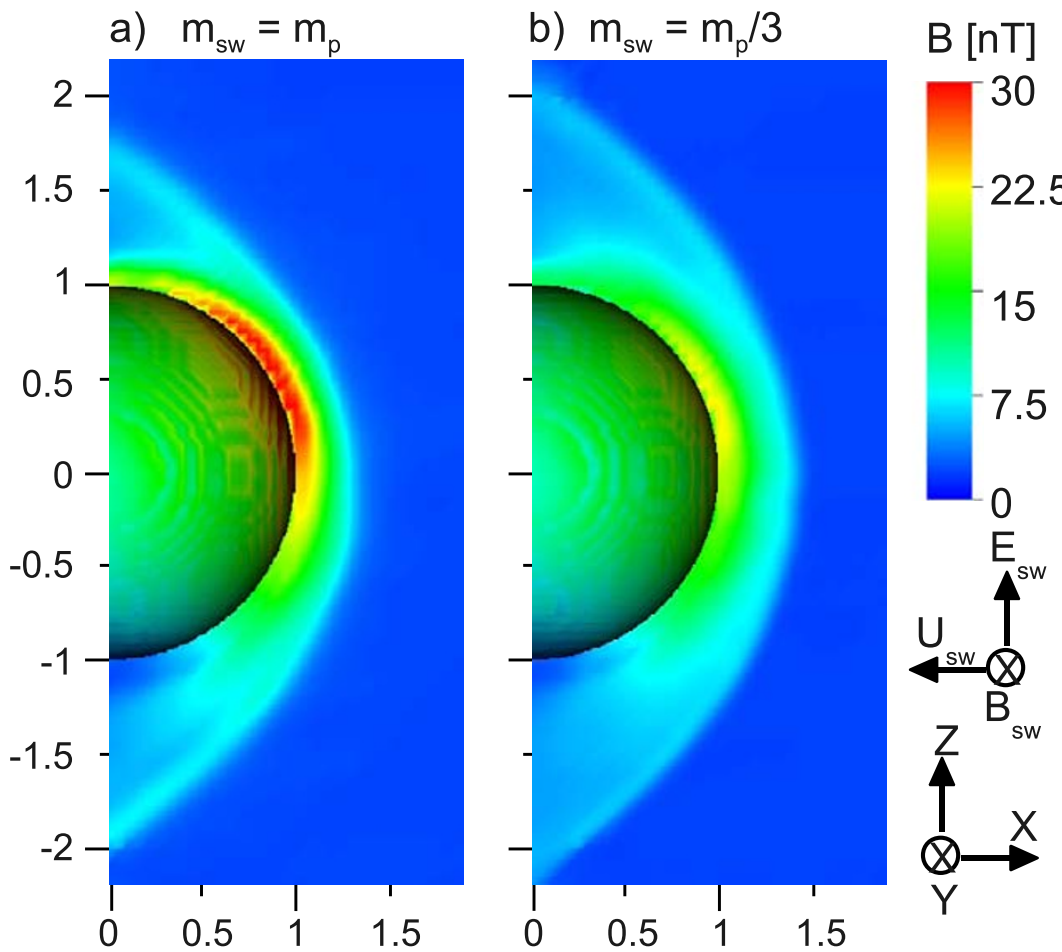

Fig. 4. The role of the mass of the solar wind positive charged ions. The figure shows the total magnetic field on the noon-midnight meridian plane $y=0$, in (a) a realistic situation, where the mass of the solar wind protons are $m_{\mathrm{p}}$ and (b) in a test case, when the mass of the solar wind positively charged ions was reduced to $1 / 3 \times m_{\mathrm{p}}$. In both runs the bulk velocity, the Alfvén velocity and the thermal velocity of the solar wind are similar The spherical shell is $3600 \mathrm{~km}\left(\sim 1.06 R_{\mathrm{M}}\right)$ from the center of the Mars and the color on its surface shows the total magnetic field. In both runs the solution is symmetric on the dawn-dusk plane, $z=0$, between the $y>0$ and $y<0$ hemispheres (figures not shown). The unit of the axes is the radius of Mars. 
barrier) near Mars. In a quantitative comparison, however, several differences can be identified. The magnetic barrier is stronger and the $\pm E_{\mathrm{sw}}$ asymmetry more pronounced in the $m_{\mathrm{p}}$ run than in the $1 / 3 \times m_{\mathrm{p}}$ run. Furthermore, the bow shock is closer to Mars in the $m_{\mathrm{p}}$ run than in the $1 / 3 \times$ $m_{\mathrm{p}}$ case. These differences imply that the asymmetries in the Martian electromagnetic environment, which affect the escape of planetary ions, are also related to the gyroradius of the solar wind ions, and not only to the sonic and Alfvén Mach numbers of the solar wind.

\section{Discussions}

This paper describes a few hybrid model test runs performed to study kinetic effects in plasma environments of Mars and Venus, especially, kinetic effects associated with the ion escape. It is shown that the hybrid model results in a 3-D electromagnetic field which includes the so called dawn-to-dusk and $\pm E_{\mathrm{sw}}$ hemisphere asymmetries (Section 3.1). The large size of the ion gyroradius results in an asymmetric ion escape pattern for heavy planetary ions at Venus (Section 3.2) and the same is also true at Mars (see, for example, Kallio et al., 2010). The newly formed ions from the Martian and Venusian neutral coronae add mass to the solar wind and the mass loading can result in asymmetries which can be seen both in plasma and magnetic field (Section 3.3). The mass of the solar wind ions and, therefore, their gyroradius affects also the electromagnetic field and its asymmetries at Mars (Section 3.4).

This brief study is, however, only a first small step in understanding the role of the kinetic effects at Mars and Venus. The test figures shown in the paper are taken from a series of test runs where various parameters were changed, such as the mass and the charge of the ions, the inner boundary conditions near the planet, the size of the planet, the form of the electric field (Hall term included/not included), upstream parameters (e.g. the strength of the IMF), the properties of the planetary ions (planetary ions included/not included, the ion production rate, the mass of planetary ions) and the grid size of the simulation. One may also anticipate that the role of the boundary condition near a planet requires special analysis, such as whether the planet is described as a super-conducting or as a slightly resistive sphere, how the ion-neutral collisions are taken into account etc. The number of free physical and numerical parameters is large, and their different combinations even larger.

A more general question is how to verify the results derived from a global model when the studied physical situation is so complicated that no analytical solution is available? This is a common situation in hybrid models and in magnetohydrodynamic (MHD) models where numerical simulations are the only possibility to solve complicated kinetic or fluid equations and electrodynamics. While comparison with data is an obvious candidate, an indication of the simulation solving the model equations correctly is if similar results are obtained also from other simulations. For example, according to the authors' knowledge, all published global hybrid model simulations for Mars and Venus result in qualitatively similar type of $\pm E_{\mathrm{sw}}$ asymmetry in the magnitude of the magnetic field as shown in Fig. 1(a). Whether the results from different models are also quantitatively similar is, however, a more difficult question. It can be addressed if different hybrid models are run with the same input parameters and the results compared. Overall, more comprehensive kinetic tests are called for in the future to evaluate the role of the kinetic effects quantitatively, such as their role in formation of the asymmetries, in the energization of the planetary ions and in the dynamical processes (waves and instabilities).

\section{Summary}

A global hybrid model was used to study kinetic effects at Mars and Venus, especially associated with the escape of planetary ions. The finite ion gyroradius effects were found to result in asymmetric global electromagnetic field, planetary ion escape regions and ion energization.

Acknowledgments. The authors thank Dr. Tero Siili for the careful reading of the manuscript. Figures 1 and 3-4 were made using the VisIt open source visualization tool. The authors would like also to thank the referees for their critical comments concerning the validation of numerical simulations.

\section{References}

Barabash, S. et al., The loss of ions from Venus through the plasma wake, Nature, 450, 650-653, doi:10.1038/nature06434, 2007.

Jarvinen, R., E. Kallio, S. Dyadechkin, P. Janhunen, and I. Sillanpää, Widely different characteristics of oxygen and hydrogen ion escape from Venus, Geophys. Res. Lett., 37, L16201, doi:10.1029/2010GL044062, 2010a.

Jarvinen, R., E. Kallio, P. Janhunen, V. Pohjola, and I. Sillanpää, Grid convergence of the HYB-Venus hybrid simulation, ASP Conf. Ser., 429, 2010b.

Kallio, E., R. Jarvinen, and P. Janhunen, Venus-solar wind interaction: Asymmetries and the escape of $\mathrm{O}^{+}$ions, Planet. Space Sci., 54(13-14), 1472-1481, 2006.

Kallio, E., K. Liu, R. Jarvinen, V. Pohjola, and P. Janhunen, Oxygen ion escape at Mars in a hybrid model: High energy and low energy ions, Icarus, 206, 152-163, doi:10.1016/j.icarus.2009.05.015, 2010.

Ledvina, S. A., Y.-J. Ma, and E. Kallio, Modeling and simulating flowing plasmas and related phenomena, Space Sci. Rev., doi:10.1007/s11214008-9384-6, 2008.

Luhmann, J. G., S. A. Ledvina, J. G. Lyon, and C. T. Russell, Venus $\mathrm{O}^{+}$ pickup ions: Collected PVO results and expectations for Venus Express, Planet. Space Sci., 54, 1457-1471, doi:10.1016/j.pss.2005.10.009, 2006.

Omidi, N. and D. Winske, A kinetic study of solar wind mass loading and cometary bow shocks, J. Geophys. Res., 92(12), 13,409-13,426, 1987.

Zhang, T. L., W. Baumjohann, J. Du, R. Nakamura, R. Jarvinen, E. Kallio, A. M. Du, M. Balikhin, J. G. Luhmann, and C. T. Russell, Hemispheric asymmetry of the magnetic field wrapping pattern in the Venusian magnetotail, Geophys. Res. Lett., 37, L14202, doi:10.1029/2010GL044020, 2010 .

E. Kallio (e-mail: esa.kallio@fmi.fi) and R. Jarvinen 